\title{
Do trialists endorse clinical trial registration? Survey of a Pubmed
}

\section{sample}

\author{
Ludovic Reveiz*1,4, Karmela Krleža-Jerić², An-Wen Chan ${ }^{3}$ and Sylvia De \\ Aguiar $^{4}$
}

Address: ${ }^{1}$ Sanitas Research Institute, Fundación Universitaria Sánitas, Bogotá, Colombia, ${ }^{2}$ Canadian Institutes of Health Research (CIHR), Ottawa, Ontario, Canada, ${ }^{3}$ Department of Medicine, University of Toronto, Toronto, Ontario, Canada and ${ }^{4}$ Latin American Ongoing Clinical Trial Register (LATINREC), Bogotá, Colombia

Email: Ludovic Reveiz* - lureveiz@colsanitas.com; Karmela Krleža-Jerić - KKrleza-Jeric@cihr-irsc.gc.ca; An-

Wen Chan - anwen.chan@utoronto.ca; Sylvia De Aguiar - sylvia_deaguiar@hotmail.com

* Corresponding author

Published: 23 October 2007

Trials 2007, 8:30 doi:10.1186/1745-62/5-8-30

This article is available from: http://www.trialsjournal.com/content/8/I/30

(c) 2007 Reveiz et al; licensee BioMed Central Ltd.

This is an Open Access article distributed under the terms of the Creative Commons Attribution License (http://creativecommons.org/licenses/by/2.0), which permits unrestricted use, distribution, and reproduction in any medium, provided the original work is properly cited.

\begin{abstract}
Introduction: Despite intense interest in trial registration, there is a wide gap between theoretical postulates on trial registration and its implementation worldwide.
\end{abstract}

Objective: We aimed to evaluate trialists views about current international guidelines on trial registration, including the World Health Organization's (WHO) International Clinical Trials Registry Platform (ICTRP) policies and the Ottawa Statement, as well as their intention to register any future clinical trials they conduct.

Methods: We identified all 40,I 58 PUBMED-indexed clinical trials published from May 2005 to May 2006 using an advanced search strategy. From a random sample of 500 confirmed clinical trials, corresponding authors with e-mail contact addresses were surveyed.

Results: A total of 275 (60\%) questionnaires from 45 countries were completed. $31 \%$ of the respondents had received only nonindustry funding during the past ten years, while $5 \%$ and $61 \%$ had received only industry or mixed funding respectively. Approximately two third of participants supported registration of all $20 \mathrm{WHO}$ Data Set items, and endorsed the Ottawa Statement part I and part 2. Delayed public disclosure of some essential data in instances where they may be considered sensitive for competitive commercial reasons was supported by $30 \%$ of the participants, whereas immediate disclosure was supported by $53 \%$. Only $21 \%$ of participants had registered all of their ongoing trials since 2005 , while $47 \%$ stated that they would provide the $20 \mathrm{WHO}$ Data Set items to a publicly accessible register for all their future clinical trials; a significantly higher proportion of participants who received only nonindustry funding $(62 \%)$ was found among those who would always provide the $20 \mathrm{WHO}$ items for future trials, compared to $42 \%$ of participants who received mixed or only industry funding. Among those who were undecided about endorsing registration. One third of participants expressed a lack of sufficient knowledge as the primary reason.

Conclusion: Although disagreement was apparent on certain issues, our findings illustrate that trial registration is gradually becoming part of the current research paradigm internationally. Our results also suggest that researchers require more knowledge to inform their decision to comply with the International standards at this early stage of voluntary trial registration. 


\section{Background}

Over the past few years, several key initiatives have enabled substantial progress in the public registration of clinical trials worldwide. Major registers such as ClinicalTrials.gov and Controlled Clinical trials-ISRCTN have been recording trial protocol information since 2000, and the number of registered trials increased dramatically in 2005 after the requirement for registration was introduced by several medical journals, led by the International Committee of Medical Journal Editors (ICMJE) [1,2]. The World Health Organization's International Clinical Trials Registry Platform (WHO ICTRP) is currently leading international efforts to implement global standards for trial registration and results reporting [24]. Based on both ethical and scientific reasons for trial registration, WHO is urging investigators, research institutions and companies to register all trials that prospectively test the effects of healthcare interventions on humans.

Furthermore, the Ottawa Group, an international group of individuals and organizations from the international medical research community, developed two consensus documents - the Ottawa Statement Part 1 on the principles of trial registration, and the Ottawa Statement Part 2 on the principles of its implementation The Ottawa group supported the WHO initiative as a good initial step, but called for more details of trial protocols to be prospectively registered [5-7]. The Institute of Medicine report endorses the ICJME requirement to prospectively register clinical trials as a condition for publication, as well as the pharmaceutical industry's commitment to register and post clinical trials results [8].

Nevertheless, despite intense interest in this topic in recent years, there is a wide gap between theoretical postulates on trial registration and its implementation worldwide. This gap may be due to differing interests of the various stakeholders involved, including researchers, patients, pharmaceutical industry, funders, governments, registries, bio-medical journal editors, and the public [911]. One of the biggest barriers to comprehensive trial registration is the lack of awareness by researchers about the importance of the problem [9]. According to the Ottawa Statement part 1, "the principal investigator has a responsibility to ensure that the sponsor(s) obtains a Unique Identification number and registers his or her contact information, the protocol information and the trial results" [5].

Although there is general agreement about the type of protocol information that should be registered for a trial, as defined by the 20-item WHO Registration Data Set (Table 1) $[11,1]$, controversy surrounded issues related to registration of key versus all secondary outcomes, as well as immediate versus delayed disclosure of 5 protocol items that were felt to be commercially sensitive by industry for some trials (study interventions, scientific title, sample size, primary and secondary outcomes) [10]. Industry has offered, instead, to consign these five hidden items to a locked electronic depository that is publicly inaccessible until the information is no longer deemed commercially sensitive [10]. Regarding results reporting on trial registration, the principles expressed in the Ottawa Statement 1 read "At a minimum, results for outcomes and analyses specified in the protocol (as approved by the IRB/IEC), as well as data on harms, should be registered regardless of whether or not they are published. If a trial is terminated prematurely, any available results should be registered along with the reason for termination." [5,6]. However, the Ottawa Group stated that researchers should have sufficient time to publish their findings before the registered results are released for public [5].

The aim of our study is to evaluate trialists' views about the global standards proposed by WHO and the Ottawa Statements, as well as their intention to register any future clinical trials they conduct.

\section{Materials and methods}

We used PUBMED to identify a sample of investigators authoring clinical trial reports published from May 2005 to May 2006. The structured search filter included the following terms: "Randomized controlled trial" [PT] OR "controlled clinical trial" [PT] OR "randomized controlled trials" [MeSH] OR "random allocation" [MeSH] OR "double-blind method" [MeSH] OR "single-blind method" [MeSH] OR "clinical trial" [PT] OR "clinical trials" [MeSH] OR "clinical trial" [TW] OR "randomized trial" [TW] OR "randomised trial" [TW]. The strategy focused on humans studies and was not limited by language or type of intervention.

For this exploratory survey, we identified 40,158 references, from which a random sample of 500 clinical trials with e-mail contact addresses was selected. Titles and abstracts identified from the sample were screened by one reviewer (LR). A previous study showed that approximately $25 \%$ of articles found by the search are clinical trials and cite the e-mail of one author [12]. The sample size was calculated assuming that 5\% of emails would not reach their intended recipient. In addition, Web-based surveys have variable response rates ( 9 to $96 \%$ ), depending on the topic, methods and participants $[13,14]$. The $a$ priori response rate was estimated to be $75 \%$. In the absence of previous data, we estimated the proportion of trialists who would endorse registration to be 50\%, yielding a sample size of 500 with a $95 \%$ confidence interval of $-5 \%$ to $5 \%$. The primary outcomes of our study were the proportion of trialists endorsing the following principles: trial registration overall, registration of all secondary out- 
Table I: Minimum data set that should be recorded for clinical trial registration, according to the International Standards launched by the World Health Organization, in 2006 (items felt to be commercially sensitive are highlighted)

\begin{tabular}{|c|c|c|}
\hline Number & Item & Abbreviated Definition/Explanation* \\
\hline I. & Primary register trial number & $\begin{array}{l}\text { Name of Primary Register, and the unique ID number assigned by the Primary } \\
\text { Register to this trial. }\end{array}$ \\
\hline 2. & Trial registration date & Date when trial was officially registered in the Primary Register. \\
\hline 3. & Secondary IDs & Other identifying numbers and issuing authorities besides the Primary Register, if any \\
\hline 4. & Source(s) of monetary or material support & Major source(s) of monetary or material support for the trial \\
\hline 5. & Primary sponsor & $\begin{array}{l}\text { The individual, organization, group or other legal entity which takes responsibility for } \\
\text { initiating, managing and/or financing a study. }\end{array}$ \\
\hline 6. & Secondary sponsor(s) & $\begin{array}{l}\text { Additional individuals, organizations or other legal persons, if any, that have agreed } \\
\text { with the primary sponsor to take on responsibilities of sponsorship. }\end{array}$ \\
\hline 7. & Contact for public queries & $\begin{array}{l}\text { Email address, telephone number, or postal address of the contact who will respond } \\
\text { to general queries, including information about current recruitment status }\end{array}$ \\
\hline 8. & Contact for scientific queries & $\begin{array}{l}\text { Email address, telephone number, or postal address, and affiliation of the person to } \\
\text { contact for scientific queries about the trial }\end{array}$ \\
\hline 9. & Public title (of the study) & Title intended for the lay public in easily understood language. \\
\hline 10. & Scientific title & $\begin{array}{l}\text { Scientific title of the study as it appears in the protocol submitted for } \\
\text { funding and ethical review }\end{array}$ \\
\hline II. & Countries of recruitment & $\begin{array}{l}\text { The countries from which participants will be, are intended to be, or have been } \\
\text { recruited. }\end{array}$ \\
\hline 12. & Health condition or problems studied & Primary health condition(s) or problem(s) studied \\
\hline 13. & Intervention(s) & $\begin{array}{l}\text { Specific name of the intervention(s) and the comparator/control(s) being } \\
\text { studied }\end{array}$ \\
\hline 14. & Key inclusion and exclusion criteria & Inclusion and exclusion criteria for participant selection, including age and sex. \\
\hline \multirow[t]{2}{*}{15.} & Study type & $\begin{array}{l}\text { A single arm study is one in which all participants are given the same intervention. } \\
\text { Trials in which participants are assigned to receive one of two or more interventions } \\
\text { are NOT single arm studies. Crossover trials are NOT single arm studies. }\end{array}$ \\
\hline & & $\begin{array}{l}\text { A trial is "randomized" if participants are assigned to intervention groups using a } \\
\text { method based on chance (e.g., random number table, random computer-generated } \\
\text { sequence, minimization, adaptive randomization). }\end{array}$ \\
\hline 16. & $\begin{array}{l}\text { Date of the first enrollment (anticipated or } \\
\text { actual date of the enrollment of the first study } \\
\text { participant) }\end{array}$ & $\begin{array}{l}\text { If the trial is being registered after recruitment of the first participant record actual } \\
\text { date of Anticipated date of enrollment of the first participant. }\end{array}$ \\
\hline I7. & Target sample size & Number of participants that this trial plans to enroll. \\
\hline 18. & Recruitment status & Recruitment status of the trial. \\
\hline 19. & Primary outcome(s) & $\begin{array}{l}\text { Outcomes are events, variables, or experiences that are measured } \\
\text { because it is believed that they may be influenced by the intervention. The } \\
\text { Primary Outcome should be the outcome used in sample size } \\
\text { calculations, or the main outcome(s) used to determine the effects of the } \\
\text { intervention(s). }\end{array}$ \\
\hline 20. & Key secondary outcomes & $\begin{array}{l}\text { Secondary outcomes are events, variables, or experiences that are of } \\
\text { secondary interest or that are measured at timepoints of secondary } \\
\text { interest. }\end{array}$ \\
\hline
\end{tabular}

\footnotetext{
* Full items definition and/or explanation can be viewed in the International Clinical Trials Registry Platform (ICTRP) website (25).
}

comes, immediate public disclosure of registered protocol items, and public disclosure of trial results.

The survey was piloted on 50 participants. For the full sample, each participant was sent a survey by email containing links to the WHO ICTRP and the Ottawa Group websites [see Additional file 1]. Up to four reminders were sent. We collected the following information: country of origin, gender, age, type of funding for clinical trials over the past 10 years, main research institution, number of ongoing trials, proportion of ongoing trials registered in public databases, knowledge and support for the WHO Registration Data Set, as well as attitudes towards the Ottawa Statements (part 1 and part 2), registration of key versus all secondary outcomes, timing of public disclosure of protocol items, and disclosure of trial results.

Data were analyzed using SPSS 12.0. Chi-square tests were used to determine associations between categorical variables.

\section{Results}

Demographic data

Out of 500 e-mail questionnaires, forty-two (8\%) email addresses were invalid (bounced back or out-of-office autoreply). We received 275/458 (60\%) replies from 45 countries. The median age of participants was 45 years (10th-90th percentiles: $33-57$ ) and 70\% were men. The 
largest number of respondents was from the United States (15\%), Italy (9\%), United Kingdom (6\%), France (5\%) and Germany (5\%). However $20 \%$ of participants did not include their country of origin in the survey, which made the evaluation of response rate by country difficult.

Of the 275 (60\%) survey respondents, 31\% received only non-industry funding over the past ten years (Table 2). Type of funding reported by participants was significantly different between women and men; $44 \%$ of women received non industry funding compared to $27 \%$ of men while $50 \%$ and $68 \%$ received mixed funding and $6 \%$ and $5 \%$ received only industry funding respectively $\left(\mathrm{X}^{2}=7.82\right.$; DF 2, $\mathrm{p}=0.02)$. Most respondents were based at an academic or hospital institution (Table 2).

\section{Trial registration issues}

One third of trialists stated that they were experts or very knowledgeable about issues related to trial registration, while 56\% reported having mild to moderate knowledge; $12 \%$ reported having no relevant knowledge.

$64 \%$ of investigators supported registration of all 20 items of the WHO Data Set, while 6\% did not support any. 65\% and $63 \%$ already had or planned to endorse the Ottawa Statements (part 1 and part 2) respectively; 3\% and 3\% did not support the statements, while $31 \%$ and $34 \%$ respectively were undecided. The attitudes of respondents were similar regarding the Ottawa Statements and the WHO standards.

Ninety-five trialists (35\%) believed that all secondary trial outcomes should be registered and disclosed, whereas $139(51 \%)$ stated that only key secondary outcomes should be registered and disclosed. With regards to timing of disclosure for five essential data items (Scientific title, Intervention(s), Target sample size, Primary outcome(s), and Key secondary outcomes) in instances where they may be considered sensitive for competitive reasons, no delayed disclosure was supported by $53 \%$ of trialists while $31 \%$ supported delayed disclosure Among those who agreed with delayed disclosure in some cases, most considered that undisclosed items should be made publicly available upon trial completion or upon regulatory approval (Figure 1). The Ottawa Group position with regards to registration of trial results was completely or mostly supported by $61 \%$ of participants, while $10 \%$ were not supportive at all.

The median number of ongoing clinical trials per respondent was 3 (10th-90th percentile range: $0-7$ ). Only $21 \%$ of participants had registered all of their ongoing trials. In addition only $47 \%$ of respondents stated that they would provide all 20 items of the WHO Data Set to a publicly accessible register for future clinical trials.

Between respondents receiving only non-industry funding compared to those receiving only industry or mixed funding, there were no significant differences for the proportion endorsing trial registration overall, registration of secondary outcomes, delayed disclosure of protocol items, or results registration. A significantly higher proportion of participants who received only nonindustry funding (62\%) was found among those who would always provide the $20 \mathrm{WHO}$ items for future trials, compared to $42 \%$ of participants who received mixed or only industry funding $\left(\mathrm{X}^{2}=8.59 ; \mathrm{df} 1, \mathrm{p}=0.0034\right)$.

$57 \%$ of participants did not provide their names; no significant difference regarding registration of future trials was found among anonymous respondents compared to those who included their names $\left(\mathrm{X}^{2}=12.4\right.$; $\mathrm{df} 7, \mathrm{p}=$ $0.087)$. However more women $(70 \%)$ were anonymous compared to men $(47 \%)\left(\mathrm{X}^{2}=6.03 ; \mathrm{df} 1, \mathrm{p}=0.014\right)$.

\section{Summary of trialists' concerns regarding trial registration}

We received a wide range of open comments. Common concerns were expressed related to (a) the lack of time to

Table 2: Characteristics of respondents $\mathbf{N}=\mathbf{2 7 5}$

\begin{tabular}{|c|c|c|c|c|}
\hline & & Total & $\begin{array}{l}\text { Women } \\
n=82 \\
30 \%\end{array}$ & $\begin{array}{l}\text { Men } \\
n=193 \\
70 \%\end{array}$ \\
\hline \multirow{4}{*}{$\begin{array}{l}\text { Source of trial funding received over } \\
\text { the past ten years }\end{array}$} & Only nonindustry & $85(31 \%)$ & $44 \%$ & $27 \%$ \\
\hline & Only industry & $14(5 \%)$ & $6 \%$ & $5 \%$ \\
\hline & Mixed & $168(61 \%)$ & $50 \%$ & $68 \%$ \\
\hline & No response & $8(3 \%)$ & & \\
\hline \multirow[t]{6}{*}{ Main institution of respondents } & Academic & $154(56 \%)$ & & \\
\hline & Hospital & 90 (33\%) & & \\
\hline & Governmental & $6(2 \%)$ & & \\
\hline & Industry (eg. pharmaceutical, device, biologics, etc) & $3(1 \%)$ & & \\
\hline & Other type & $14(5 \%)$ & & \\
\hline & No response & $8(3 \%)$ & & \\
\hline
\end{tabular}




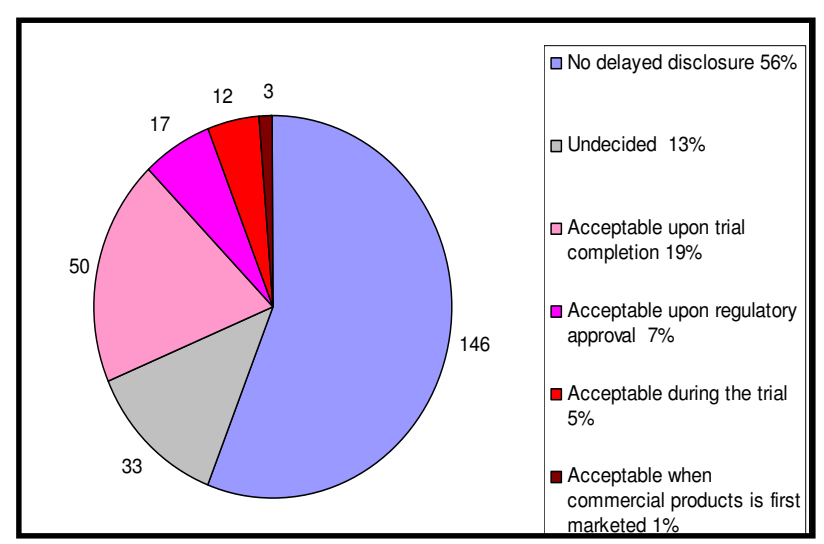

Figure I

Position of respondents regarding a delayed disclosure of five essential protocol items $\mathrm{n}=26 \mathrm{I}$.

complete "bureaucratic" tasks (4\%), (b) the possibility that registration of early (phase I) trials could deter research as their results may be misinterpreted (2\%), and (c) that the results of poorly-designed trials might be confusing (1\%). Three trialists expressed concern that the Ottawa Statement or the WHO initiative offers no protection from other competing investigators who may copy the trial, recruit faster and publish sooner. In addition, they mentioned the administrative burden of handling additional public queries as well as contractual obligations to sponsors would be difficult to handle. Other participants stated that some countries have limited internet access, and that registration should be a tool for protecting the patient's rights rather than yet another means of overburdening researchers.

\section{Discussion \\ Main findings}

Our survey provides useful information about trialists' attitudes towards clinical trial registration. Although more than $60 \%$ of respondents support both the WHO 20-item Data Set and the Ottawa Statements (parts 1 and 2), only $47 \%$ declared that they would always provide the WHO Data Set to a publicly accessible register for future clinical trials. In addition a third of participants were undecided regarding trial registration while a small minority rejected it outright.

The proposal by WHO and the Ottawa Group to submit protocol information at trial inception as well as results to a freely accessible public register is a crucial step towards promoting research transparency. However, countries have different legal requirements and most do not require public registration of all clinical trials. They also have diverse levels of public disclosure of information. For example all entities conducting clinical trials of experi- mental treatments for serious or life-threatening conditions or diseases in the United States (US) are required to submit certain information to the ClinicalTrials.gov register, which is a publicly accessible database. However the US legislation does not include other type of diseases, and does not require the registration of all 20 items suggested by WHO. Furthermore, information on Phase I trials of drugs and devices is not publicly available unless they have been approved by the US Food and Drug Administration [15]. It is evident that there is a need for comprehensive legislation on trial registration. Otherwise, when ongoing or completed trials remain hidden, researchers may be unknowingly and inappropriately duplicating trials on similar interventions that had already been shown in previous trials to have serious adverse events or no benefit.

Our survey demonstrated greater support for immediate rather than delayed disclosure of information submitted to trial registers ( $53 \%$ vs. $30 \%$ ), Figure 1 . However, some researchers argue that confidentiality issues and contractual obligations with sponsors would be difficult to handle if they decided to register all clinical trials in a publicly accessible register. Some stakeholders also claim that disclosure of all $20 \mathrm{WHO}$ data set items may sometimes endanger proprietary rights. However others argue that delayed disclosure would facilitate registration of incomplete information for a given trial, and hidden trial information would not meet ethical and scientific standards [4,16-18]. In addition, trial participants are already informed about a given study as part of the informed consent process, and intelligence companies provide detailed data about pipeline drugs at a cost; thus this information is not secret $[19,20]$. Furthermore, informed choice about which particular trial to join requires that information about all ongoing trials be available to each potential trial participant.

A universal requirement for clinical trial registration as a condition of ethics approval would level the playing field and address concerns over competitive disadvantage [21]. In the interim, voluntary registration of clinical trial information remains an important first step, and dissemination strategies to inform researchers about trial registration are thus needed, particularly in developing countries.

More participants supported registration and disclosure of only key rather than all secondary outcomes. Use of the adjective "key" introduces subjectivity in its definition, as outcomes may be considered as non-key by some researchers but essential by others. 


\section{Limitations}

Some concerns have been raised regarding Web based surveys including coverage bias or bias due to sampled individuals not having or choosing not to access the Internet [22-24]. There was selection bias due to less than half of the authors having e-mail addresses listed in the publication. For example there were 8 publications from Russia but no electronic addresses were available. Furthermore a substantial percentage of those with valid email addresses did not respond. There are also limitations related to PubMed. Although the MEDLINE database indexes more than 3500 biomedical journals, it excludes many existing trials and authors worldwide. In addition PUBMED may be biased toward English-language journals and has poorer coverage of European journals than compared to other databases. Considering that $74 \%$ of respondents were from non-English speaking countries, there was unlikely to be language bias from the survey being in English.

It should also be noted that there were few participants from commercial or government institutions.

At the same time, participants who had some knowledge about trial registration may have been more able to respond. It is highly probable that a number of respondents may have needed more knowledge about trial registration to answer specific questions, although we provided links to relevant information on the Internet.

It is disappointing that although $64 \%$ of participants endorsed trial registration, only $47 \%$ stated that they would always provide at least all 20 WHO Data Set items for future trials. This finding may reflect their concerns over academic or commercial interests.

\section{Conclusion}

Selective reporting of information about ongoing and completed trials is harmful for society as it violates ethical and scientific responsibilities, and distorts the body of evidence available for clinical decision-making. Voluntary registration of clinical trial information is an important and complex initial step that has the support of almost half of trialists. Dissemination strategies to inform researchers about the process and benefits of trial registration are needed to improve compliance.

\section{Competing interests}

Ludovic Reveiz is currently the Director of the Latin American Clinical Trial Register (Latinrec), which is part of a non profit Organization that aim to freely register clinical trials in Latin America. Karmela Krleza-Jeric is leading the Ottawa group and is a member of the Scientific Advisory Board (SAG) of the WHO International Clinical Trials Registry Platform (ICTRP). An-Wen Chan is a member of the Ottawa Group editorial and was formerly a scientist with the ICTRP, WHO.

\section{Authors' contributions}

All the authors conceived the study and participated in the design. AWC, KK and LR contributed to the questionnaire design. SA administrated electronic questionnaires and entered the data. LR analyzed the data. All authors contributed to drafting the manuscript and read and approved the final manuscript.

\section{Additional material}

\section{Additional file 1}

Survey. Survey provided to respondents.

Click here for file

[http://www.biomedcentral.com/content/supplementary/17456215-8-30-S1.doc]

\section{Acknowledgements}

The authors thank Enrique Hernandez for his technical assistance for performing the Web Questionnaire.

\section{References}

I. De Angelis CD, Drazen JM, Frizelle FA, Haug C, Hoey J, Horton R, Kotzin S, Laine C, Marusic A, Overbeke AJ, Schroeder TV, Sox HC, Van Der Weyden MB: International Committee of Medical Journal Editors. Is this clinical trial fully registered? A statement from the International Committee of Medical Journal Editors. Ann Intern Med 2004, I41:477-8 [http://www.icmje.org/ clin trialup.htm]. Accessed 19 February 2007

2. DeAngelis C, Drazen JM, Frizelle FA, Haud C, Hoey J, Horton R, et al: Clinical trial registration: a statement from the International Committee of Medical Journal Editors. N Engl J Med 2004, 35 I: I 250-I.

3. World Health Organization (2005) World Health Organization international clinical trials registry platform: New standards for registration of human medical research [http://www.who.int/ictrp/en/]. Geneva: World Health Organization Accessed 17 October 2006

4. Sim I, Chan AW, Gulmezoglu AM, Evans T, Pang T: Clinical trial registration: transparency is the watchword. Lancet 2006, 367(9523): $1631-3$.

5. Krleza-Jeric K, Chan AW, Dickersin K, Sim I, Grimshaw J, Gluud C, (for the Ottawa group): Principles for international registration of protocol information and results from human trials of health related interventions: Ottawa statement (part I). BMJ 2005, 330(7497):956-8.

6. The Ottawa Group: The Ottawa Statement, Part one: Principles for international registration of protocol information and results from human trials of health related interventions. [http://ottawagroup.ohri.ca/]. Accessed 17 October 2006

7. The Ottawa Group: Draft - The Ottawa Statement, Part Two: Principles of operationalisation for international trial registration. N Engl J Med. 2005 Jun 9 352(232436-8 [http://ottawa group.ohri.ca/statement2.html]. Accessed 17 October 2006

8. Committee on the Assessment of the US Drug Safety System: The Future of Drug Safety: Promoting and Protecting the Health of the Public. [http://www.nap.edu/catalog//1750.html\#toc]. Accessed in June 2007

9. Dickersin K, Rennie D: Registering Clinical Trials. JAMA 2003, 290:516-523.

10. KrleŽa-Jerić K: Clinical Trial Registration: The Differing Views of Industry, the WHO, and the Ottawa Group. PLoS Med 2005, 2(II):e378. 
II. Reveiz L, Delgado MB, Urrutia G, Ortiz Z, Garcia Dieguez M, MartiCarvajal A, et al:: The Latin American Ongoing Clinical Trial Register (LATINREC). Rev Panam Salud Publica 2006, 19(6):417-22.

12. Reveiz L, Cardona AF, Ospina EG, de Aguiar S: An e-mail survey identified unpublished studies for systematic reviews. J Clin Epidemiol 2006, 59(7):755-8.

13. Braithwaite D, Emery J, De Lusignan S, Sutton S: Using the Internet to conduct surveys of health professionals: a valid alternative? Fam Pract 2003, 20(5):545-5I.

14. Eysenbach $G$, Wyatt J: Using the Internet for surveys and health research. J Med Internet Res 2002, 4(2): EI3.

15. Turner EH: A Taxpayer-Funded Clinical Trials Registry and Results Database. PLoS Med 2004, I(3):e60.

16. Lemmens T, Bouchard R: Comments on the Legal, Regulatory and Ethical Aspects of the WHO Clinical Trial Registry Platform. Submitted as part of the Formal Consultation on Disclosure Timing Policy. [http://www.who.int/ictrp/ 01I Lemmens Bouchard 5April06.pdf]. Accessed in November 2006

17. Laine C, Horton R, DeAngelis C, Drazen J, Frizelle F, Godlee F, Haug C, Hebert P, Kotzin S, Marusic A, Sahni P, Schroeder T, Sox H, Van der Weyden M, Verheugt F: International Committee of Medical Journal Editors (ICMJE). Clinical trial registration: looking back and moving ahead. N Engl J Med 2007, 356(26):2734-6.

18. Gotzsche P: Disclosures should not be delayed. [http:// www.who.int/ictrp/006-Peter Gotzsche 31March06.pdf]. Accessed in November 2006

19. Pharmaceutical R\&D Pipeline News [http://www.Pharm aprojects.com]

20. BioPharm Insight [http://www.infinata5.com/biopharmfree]

21. Godlee F: An international standard for disclosure of clinical trial information. BMJ 2006, 332: I 107-1 108.

22. Eysenbach $G, W y a t t ~]$ : Using the Internet for surveys and health research. J Med Internet Res 2002, 4(2):EI3.

23. Schleyer TK, Forrest JL: Methods for the design and administration of web-based surveys. J Am Med Inform Assoc 2000, 7(4):416-25.

24. Edwards P, Roberts I, Clarke M, DiGuiseppi C, Pratap S, Wentz R, Kwan I, Cooper R: Methods to increase response rates to postal questionnaires. Cochrane Database of Systematic Reviews 2007. Art. No.: MR000008.DOI: 10.1002//465/858

25. International Clinical Trials Registry Platform (ICTRP) [http://www.who.int/ictrp/data set/en/]

Publish with Biomed Central and every scientist can read your work free of charge

"BioMed Central will be the most significant development for disseminating the results of biomedical research in our lifetime. "

Sir Paul Nurse, Cancer Research UK

Your research papers will be:

- available free of charge to the entire biomedical community

- peer reviewed and published immediately upon acceptance

- cited in PubMed and archived on PubMed Central

- yours - you keep the copyright
BiolMedcentral 